PROCEEDINGS OF THE

AMERICAN MATHEMATICAL SOCIETY

Volume 124, Number 12, December 1996, Pages 3595-3604

S 0002-9939(96)03442-9

\title{
INTEGER-VALUED POLYNOMIALS ON KRULL RINGS
}

\author{
SOPHIE FRISCH
}

(Communicated by Wolmer V. Vasconcelos)

\begin{abstract}
If $R$ is a subring of a Krull ring $S$ such that $R_{Q}$ is a valuation ring for every finite index $Q=P \cap R, P$ in $\operatorname{Spec}^{1}(S)$, we construct polynomials that map $R$ into the maximal possible (for a monic polynomial of fixed degree) power of $P S_{P}$, for all $P$ in $\operatorname{Spec}^{1}(S)$ simultaneously. This gives a direct sum decomposition of $\operatorname{Int}(R, S)$, the $S$-module of polynomials with coefficients in the quotient field of $S$ that map $R$ into $S$, and a criterion when $\operatorname{Int}(R, S)$ has a regular basis (one consisting of 1 polynomial of each non-negative degree).
\end{abstract}

\section{INTRODUCTION}

If $A$ is an infinite subset of a domain $S$, we write $\operatorname{Int}(A, S)$ for the $S$-module of polynomials with coefficients in the quotient field of $S$ that - when acting as a function by substitution of the variable - map $A$ into $S$. For $\operatorname{Int}(S, S)$, the ring of integer-valued polynomials on $S$, we write $\operatorname{Int}(S)$. Beyond the fact (known of old) that the binomial polynomials $\left(\begin{array}{l}x \\ n\end{array}\right)=\frac{x(x-1) \ldots(x-n+1)}{n !}$ form a basis of the free $\mathbb{Z}$-module $\operatorname{Int}(\mathbb{Z})$, the study of $\operatorname{Int}(S)$ originated with Pólya [16] and Ostrowski [15], who let $S$ be the ring of integers in a number field (their results have been generalized to Dedekind rings by Cahen [4]). $\operatorname{Int}(R, S)$ for $R \neq S$ has only begun to attract attention more recently [2], [3], [6], [8], [11], [13].

We will treat Pólya's and Ostrowski's questions in the case where $R \neq S$ and $S$ is a Krull ring; in particular the question when $\operatorname{Int}(R, S)$ is a free $S$-module that admits a regular basis, and the related one of determining the highest power of $P S_{P}$, where $P$ is a height 1 prime ideal of $S$, that a monic polynomial of fixed degree can map $R$ into. Following Pólya, we call a sequence of polynomials $\left(g_{n}\right)_{n \in \mathbb{N}_{0}}$ regular, if $\operatorname{deg} g_{n}=n$ for all $n$. One basic connection between a module of polynomials and the modules of leading coefficients should be kept in mind:

0.1 Lemma. Let $R$ be a unitary subring of a field $K, M$ an $R$-submodule of $K[x]$, and $I_{n}=\{$ leading coefficients of $n$-th degree polynomials in $M\} \cup\{0\}$.

(i) If $\left(g_{n}\right)_{n \in \mathbb{N}_{0}}$ is a regular sequence of monic polynomials in $K[x]$ such that $I_{n} g_{n} \subseteq M$ for all $n$, then $M=\sum_{n=0}^{\infty} I_{n} g_{n}$ (direct sum).

(ii) A regular set of polynomials in $M$ is an $R$-basis if and only if the leading coefficient of the $n$-th degree polynomial generates $I_{n}$ as an $R$-module.

(iii) $M$ has a regular $R$-basis if and only if each $I_{n}$ is non-zero and cyclic.

Received by the editors September 2, 1994 and, in revised form, May 1, 1995.

1991 Mathematics Subject Classification. Primary 13B25, 13F05; Secondary 13F20, 11C08.

(C)1996 American Mathematical Society 
Proof. (i) If $\left(g_{n}\right)_{n \in \mathbb{N}_{0}}$ is as stated, then $\sum_{n=0}^{\infty} I_{n} g_{n} \subseteq M$ and the sum is direct, since $\operatorname{deg}\left(g_{n}\right)=n$ makes the $g_{n}$ linearly independent over $K$. An induction on $N=\operatorname{deg} f$ shows that $f \in M$ implies $f \in \sum_{n=0}^{N} I_{n} g_{n}$. Indeed, for $N=0, f \in I_{0}=g_{0} I_{0}$, and if $N>0$ and $a_{N}$ is $f$ 's leading coefficient, then $a_{N} \in I_{N}$, so $h=f-a_{N} g_{N} \in M$ and $h \in \sum_{n=0}^{N-1} I_{n} g_{n}$ by induction hypothesis. (ii) and (iii) are easy.

\section{Polynomials mapping A Set into A Discrete VAluation Ring}

Throughout section one, $v$ is a discrete valuation on a field $K$ with value-group $\Gamma_{v}=\mathbb{Z}$ and $v(0)=\infty$, and $R_{v}$ its valuation ring with maximal ideal $M_{v}$. In a kind of generic local regular basis theorem, we will establish the connection (wellknown in special cases) between $\operatorname{Int}\left(A, R_{v}\right)$ and the maximal power of $M_{v}$ that a monic polynomial of degree $n$ can map $A$ into, for all $A \subseteq K$ for which this maximum exists for every $n$. A subset $A$ of the quotient field of a domain $R$ is called $R$-fractional if there exists a $d \in R \backslash\{0\}$ such that $d A \subseteq R$.

1.0 Lemma. If $R$ is an integrally closed domain with quotient field $L, A \subseteq L$ and $f$ non-constant $\in L[x]$ then $f(A)$ is $R$-fractional if and only if $A$ is.

Proof. Let $f \in L[x], \operatorname{deg} f=n>0$. If $f(A)$ is $R$-fractional there is a non-zero $d \in R$, with $d f(a) \in R$ for every $a \in A$. Let $c \in R \backslash\{0\}$, such that $c f \in R[x]$, and set $g=c d f=c_{n} x^{n}+\ldots+c_{0}$. For every $a \in A, g(a) \in R$ implies that $c_{n} a$ is integral over $R$, therefore $c_{n} a \in R$ and $c_{n} A \subseteq R$. The converse is clear.

Since a set $B \subseteq K$ is $R_{v}$-fractional if and only if $\min _{b \in B} v(b)$ exists in $\mathbb{Z} \cup$ $\{\infty\}$, Lemma 1.0 shows that $A$ being $R_{v}$-fractional is necessary and sufficient for $\min _{a \in A} v(f(a))$ to exist in $\mathbb{Z} \cup\{\infty\}$ for any non-constant $f \in K[x]$. To exclude polynomials identically zero on $A$, for which $\min _{a \in A} v(f(a))=\infty$, we need $\operatorname{deg} f<$ $|A|$, so that the conditions on $A$ in Lemma 1.1 below are necessary.

1.1 Lemma. Let $n \in \mathbb{N}_{0}$. If $A$ is an $R_{v}$-fractional subset of $K$ with $|A|>n$, then $\max \left\{\min _{a \in A} v(f(a)) \mid f\right.$ monic $\left.\in K[x], \operatorname{deg} f=n\right\}$ exists.

Proof. The case $n=0$ is trivial; so let $n>0$ and $m \in \mathbb{N}$ such that $A$ is not contained in any union of $n$ cosets of $M_{v}^{m}$ in $K$. Such an $m$ exists, since $n<|A|$ and by the Krull Intersection Theorem $\bigcap_{m \in \mathbb{N}} M_{v}^{m}=(0)$. We show that for every monic $f \in K[x]$ of degree $n$ there exists an $a_{0} \in A$ with $v\left(f\left(a_{0}\right)\right)<n m$ (and consequently $\max \left\{\min _{a \in A} v(f(a)) \mid f\right.$ monic $\left.\left.\in K[x], \operatorname{deg} f=n\right\}<n m\right)$.

Let $v^{\prime}$ be an extension of $v$ to the splitting field of $f$ over $K, R_{v^{\prime}}$ its valuationring with maximal ideal $M_{v^{\prime}}$, and $e=\left[\Gamma_{v^{\prime}}: \Gamma_{v}\right] . A$ is not contained in any union of $n$ cosets of $M_{v^{\prime}}$ me in $K^{\prime}$. Pick an $a_{0} \in A$ that is not in $u+M_{v^{\prime}}$ me for any root $u$ of $f$ in $K^{\prime}$; then $v\left(f\left(a_{0}\right)\right)=v^{\prime}\left(f\left(a_{0}\right)\right)=\sum_{i=1}^{n} v^{\prime}\left(a_{0}-u_{i}\right)<n m$.

1.2 Theorem. Let $A$ be an infinite, $R_{v}$-fractional subset of $K$. For $n \in \mathbb{N}_{0}$ set $\gamma_{v, A}(n)=\max \left\{\min _{a \in A} v(f(a)) \mid f\right.$ monic $\left.\in K[x], \operatorname{deg} f=n\right\}$.

(i) $M_{v}{ }^{-\gamma_{v}, A}(n)=\left\{\right.$ leading coefficients of degree $n$ polynomials in $\left.\operatorname{Int}\left(A, R_{v}\right)\right\} \cup$ $\{0\}$.

(ii) A regular basis of $\operatorname{Int}\left(A, R_{v}\right)$ is given by $\left(c_{n} g_{n}\right)_{n \in \mathbb{N}_{0}}$, with $g_{n} \in K[x]$ monic, $\operatorname{deg} g_{n}=n$, and $c_{n} \in K$, such that $\min _{a \in A} v\left(g_{n}(a)\right)=\gamma_{v, A}(n)$ and $v\left(c_{n}\right)=$ $-\gamma_{v, A}(n)$. 
Proof. Let $I_{n, v}=$ \{leading coefficients of degree $n$ polynomials $\left.\operatorname{in} \operatorname{Int}\left(A, R_{v}\right)\right\} \cup$ $\{0\}$. The leading coefficient $c_{n}$ of any $n$-th degree polynomial in $\operatorname{Int}\left(A, R_{v}\right)$ must satisfy $v\left(c_{n}\right) \geq-\gamma_{v, A}(n)$, so $I_{n, v} \subseteq M_{v}{ }^{-\gamma_{v, A}(n)}$. Now, for $n \in \mathbb{N}_{0}$, let $g_{n}$ be monic of degree $n$ in $K[x]$ with $\min _{a \in A} v\left(g_{n}(a)\right)=\gamma_{v, A}(n)$ (such things exist by dint of Lemma 1.1). Then $M_{v}{ }^{-\gamma_{v, A}(n)} g_{n} \subseteq \operatorname{Int}\left(A, R_{v}\right)$, so $M_{v}{ }^{-\gamma_{v, A}(n)} \subseteq I_{n, v}$. This shows (i) and also that $I_{n, v} g_{n} \subseteq \operatorname{Int}\left(A, R_{v}\right)$ for all $n \in \mathbb{N}_{0}$. (ii) follows by Lemma 0.1 and the fact that $M_{v}{ }^{-\gamma_{v, A}(n)}=c_{n} R_{v}$ for every $c_{n} \in K$ with $v\left(c_{n}\right)=-\gamma_{v, A}(n)$.

Before deriving a formula for $\max \left\{\min _{a \in A} v(f(a)) \mid f\right.$ monic $\left.\in K[x], \operatorname{deg} f=n\right\}$, when $A$ is a subring of $R_{v}$, we check that the other plausible way of normalizing the polynomials would yield the same value. We also see that polynomials mapping $A \subseteq R_{v}$ into the maximal possible power of $M_{v}$ can be chosen to split with their roots in any set that $M_{v}$-adically approximates $A$ (for instance in $A$ itself, or, if $R_{v}$ is the localization of a ring $R$ at a prime ideal of finite index, in $R$ ). We need a lemma from [7] (but include the proof).

1.3 Lemma. Let $f \in R_{v}[x]$, not all of whose coefficients lie in $M_{v}$, split over $K$, as $f(x)=d\left(x-b_{1}\right) \cdot \ldots \cdot\left(x-b_{m}\right) \cdot\left(x-c_{1}\right) \cdot \ldots \cdot\left(x-c_{l}\right)$ with $v\left(b_{i}\right)<0, v\left(c_{i}\right) \geq 0$, and put $f_{+}(x)=\left(x-c_{1}\right) \cdot \ldots \cdot\left(x-c_{l}\right)$. Then, for all $r \in R_{v}, v(f(r))=v\left(f_{+}(r)\right)$.

Proof. For $r \in R_{v} v\left(r-b_{i}\right)=v\left(b_{i}\right)$ and so $v(f(r))=v(d)+\sum_{i=1}^{m} v\left(b_{i}\right)+v\left(f_{+}(r)\right)$; we show $v(d)=-\sum_{j=1}^{m} v\left(b_{i}\right)$. Consider $d^{-1} f(x)=x^{n}+a_{n-1} x^{n-1}+\ldots+a_{0}$. Since $f \in R_{v}[x] \backslash M_{v}[x], v(d)=-\min _{0 \leq k \leq n} v\left(a_{k}\right)$. But $a_{k}$ is the elementary symmetric polynomial of degree $n-k$ in the $b_{i}$ and $c_{i}$, so the minimal valuation is attained by $v\left(a_{n-m}\right)=\sum_{i=1}^{m} v\left(b_{i}\right)$.

1.4 Proposition. Let $A \subseteq R_{v}$ and $0 \leq n<|A|$; then $\alpha$ and $\gamma$ below are equal:

$$
\begin{aligned}
& \alpha=\max \left\{\min _{a \in A} v(f(a)) \mid f \in R_{v}[x] \backslash M_{v}[x], \operatorname{deg} f=n\right\}, \\
& \gamma=\max \left\{\min _{a \in A} v(f(a)) \mid f \text { monic } \in K[x], \operatorname{deg} f=n\right\} .
\end{aligned}
$$

If, furthermore, $B \subseteq R_{v}$, such that $B$ intersects every coset of $M_{v}{ }^{l}$ that $A$ intersects, for all $l \in \mathbb{N}$, then $\delta$ below is equal to $\alpha$ and $\gamma$; and so is $\beta$, if $B$ is also a ring:

$$
\begin{aligned}
& \beta=\max \left\{\min _{a \in A} v(f(a)) \mid f \in B[x] \backslash\left(M_{v} \cap B\right)[x], \operatorname{deg} f=n\right\}, \\
& \delta=\max \left\{\min _{a \in A} v(f(a)) \mid f(x)=\prod_{i=1}^{n}\left(x-d_{i}\right), d_{i} \in B\right\} .
\end{aligned}
$$

Proof. Let $B$ be a fixed subset of $R_{v}$ that intersects every coset of every power of $M_{v}$ that $A$ intersects (e.g. $B=R_{v}$, when only interested in $\alpha$ and $\gamma$ ). For $n=0$ all four expressions are equal to 0 ; now consider a fixed $n>0$. Clearly $\delta \leq \gamma$ and, if $B$ is a ring, $\delta \leq \beta \leq \alpha$. Also $\gamma \leq \alpha$, because, given $f$ monic in $K[x]$, there exists a $d \in R_{v}$ such that $d f=g \in R_{v}[x] \backslash M_{v}[x]$ and for all $a \in A$ $v(g(a))=v(d)+v(f(a)) \geq v(f(a))$, and so $\min _{a \in A} v(g(a)) \geq \min _{a \in A} v(f(a))$.

To show $\alpha \leq \delta$, we fix $f \in R_{v}[x] \backslash M_{v}[x]$ of degree $n$ and construct a monic $g$ that splits with roots in $B$ such that $v(g(a)) \geq \min _{a \in A} v(f(a))$ for all $a \in A$. Let $v^{\prime}$ be an extension of $v$ to the splitting field of $f$ over $K$. For all $a \in A$, $v^{\prime}(f(a))=v^{\prime}\left(f_{+}(a)\right)$ with $f_{+}(x)=\prod_{i=1}^{l}\left(x-c_{i}\right)$, where the $c_{i}$ are the roots of $f$ in $R_{v^{\prime}}$, by Lemma 1.3. Put $s=\min _{a \in A} v^{\prime}\left(f_{+}(a)\right)$. We replace each $c_{i}$ by a $d_{i} \in B$ chosen such that $\prod_{i=1}^{l}\left(x-d_{i}\right)=h(x)$ satisfies $v^{\prime}(h(a)) \geq s$ for all $a \in A$. If $\left(c_{i}+M_{v^{\prime}}^{k}\right) \cap A \neq \emptyset$ for all $k \in \mathbb{N}$, we pick $d_{i}$ out of $\left(c_{i}+M_{v^{\prime}}^{s}\right) \cap B$; otherwise out of 
$\left(c_{i}+M_{v^{\prime}}^{k}\right) \cap B$ with $k$ maximal such that $\left(c_{i}+M_{v^{\prime}}^{k}\right) \cap A \neq \emptyset$. Since the intersection of a residue class of $M_{v^{\prime}}^{k}$ in $R_{v^{\prime}}$ with $R_{v}$ is either empty or an entire residue class of a power of $M_{v}$ in $R_{v}$, and $B$ intersects all of these that $A$ intersects, it is possible to find such $d_{i}$ in $B$. Now for every $a \in A$ either $v^{\prime}\left(a-d_{i}\right) \geq v^{\prime}\left(a-c_{i}\right)$ for all $i$ and so $v^{\prime}(h(a)) \geq v^{\prime}\left(f_{+}(a)\right) \geq s$, or $v^{\prime}\left(a-d_{i}\right) \geq s$ for some $i$ and hence $v^{\prime}(h(a)) \geq s$. To get a polynomial of degree $n$, set $g(x)=\left(x-d_{0}\right)^{n-l} h(x), d_{0} \in B$.

\section{Polynomials mapping into a maximal power of $M_{v}$}

If $R$ is an infinite subring of a discrete valuation ring $R_{v}$, we will construct polynomials $g_{n}(x)=\left(x-a_{1}\right) \ldots\left(x-a_{n}\right)$ that map $R$ into the maximal possible (for a monic polynomial of degree $n$ ) power of $M_{v}$, by finding sequences $\left(a_{i}\right)$ in $R$ that show a nice distribution among the cosets of $M_{v}^{n} \cap R$, to serve as roots.

This generalizes a procedure of Pólya [16] (also used by Gunji and McQuillan [12], [14], Cahen [4] and others) for the special case where $R_{v}=R_{Q}, Q$ being a prime ideal of index $q$ in $R$ such that $R_{Q}$ is a discrete valuation ring: Pick $\pi \in Q \backslash Q^{2}$ and a complete set of residues $r_{0}, \ldots, r_{q-1}$ of $Q$ in $R$ and define $a_{n}=\sum_{i \geq 0} r_{c_{i}} \pi^{i}$, if $n=\sum_{i>0} c_{i} q^{i}$ is the $q$-adic expansion of $n$. The resulting polynomials map $R$ into the highest possible power of $Q$ and can be used to give a regular basis of $\operatorname{Int}\left(R_{v}\right)$ (most clearly stated in [14]). Gilmer [10] has remarked that the construction even works for $\operatorname{Int}(D), D$ a quasi-local ring with principal maximal ideal.

The $\mathcal{I}$-sequences below are defined for any commutative ring $R$. All sequences are indexed by an initial segment of $\mathbb{N}$ or $\mathbb{N}_{0}$. Quantifiers over indices of such a sequence are assumed to range over precisely the index-set.

2.0 Definition. If $\mathcal{I}$ is a set of ideals in a commutative ring $R$, we define an $\mathcal{I}$-sequence in $R$ to be a sequence $\left(a_{n}\right)$ of elements in $R$ with the property

$$
\forall I \in \mathcal{I} \quad \forall n, m \quad a_{n} \equiv a_{m} \bmod I \quad \Longleftrightarrow \quad[R: I] \mid n-m .
$$

We define a homogeneous $\mathcal{I}$-sequence to be one with the additional property

$$
\forall I \in \mathcal{I} \quad \forall n \geq 1 \quad a_{n} \in I \Longleftrightarrow[R: I] \mid n .
$$

(Any infinite $[R: I]$ we regard as dividing 0 , but no other integer.) Note that $a_{1}, a_{2}, \ldots$ is a homogeneous $\mathcal{I}$-sequence if and only if $0=a_{0}, a_{1}, a_{2}, \ldots$ is an $\mathcal{I}$-sequence.

2.1 Proposition. Let $\mathcal{I}=\left\{I_{n} \mid n \in \mathbb{N}\right\}$ be a descending chain of ideals in a commutative ring $R$. Then there exists an infinite homogeneous $\mathcal{I}$-sequence in $R$.

Proof. Put $I_{0}=R$. For $k \geq 0$, if $\left[I_{k}: I_{k+1}\right]$ is finite, let $\left\{a_{j}^{(k)} \mid 0 \leq j<\left[I_{k}: I_{k+1}\right]\right\}$ be a system of representatives of $I_{k}: I_{k+1}$ with $a_{0}^{(k)}=0$, otherwise let $\left(a_{j}^{(k)}\right)_{j \in \mathbb{N}_{0}}$ be a sequence in $I_{k}$ of elements pairwise incongruent $\bmod I_{k+1}$, with $a_{0}^{(k)}=0$. If $I_{N} \in \mathcal{I}$ with $\left[R: I_{N}\right]$ finite, then every $n<\left[R: I_{N}\right]$ has a unique representation $n=\sum_{k=0}^{N-1} j_{k}\left[R: I_{k}\right]$ with $0 \leq j_{k}<\left[I_{k}: I_{k+1}\right]$, and we set $a_{n}=\sum_{k=0}^{N-1} a_{j_{k}}^{(k)}$. If the indices of ideals in $\mathcal{I}$ get arbitrarily large while remaining finite, this defines our $\mathcal{I}$-sequence inductively. Otherwise there exists $I_{N} \in \mathcal{I}$ of maximal finite index such that either $\left[I_{N}: I_{N+1}\right]$ is infinite or $I_{m}=I_{N}$ for $m \geq N$. Define $a_{n}$ for $n<\left[R: I_{N}\right]$ as above. Then, in the first case, set $a_{m}=a_{q}{ }^{(N)}+a_{r}$ for $m=q\left[R: I_{N}\right]+r$ with $0 \leq r<\left[R: I_{N}\right]$, and $a_{m}=a_{r}$ in the second. 
2.2 Facts. (i) For $I \in \mathcal{I}$ of finite index in $R$, any $[R: I]$ consecutive terms of an $\mathcal{I}$-sequence form a complete set of representatives of $R \bmod I$.

(ii) If $\left(a_{i}\right)_{i=1}^{n}$ is an $\mathcal{I}$-sequence in $R$ then $\left(r-a_{i}\right)_{i=1}^{n}$ is an $\mathcal{I}$-sequence for every $r \in R$ and $\left(a_{n}-a_{n-i}\right)_{i=0}^{n-1}$ is a homogeneous $\mathcal{I}$-sequence.

The following lemma will be needed for globalization.

2.3 Lemma. If $a_{1}, \ldots, a_{l}$ is an $\mathcal{I}$-sequence for a chain of ideals $\mathcal{I}, J \in \mathcal{I}$ with $[R: J]>l$, and $b_{1}, \ldots, b_{l} \in R$ such that $b_{n} \equiv a_{n} \bmod J$ for $1 \leq n \leq l$, then $\left(b_{n}\right)$ is also an $\mathcal{I}$-sequence, and homogeneous if $\left(a_{n}\right)$ is.

Proof. Let $I \in \mathcal{I}$ and $1 \leq n, m \leq l$. First suppose $n \equiv m \bmod [R: I]$. Then $n=m$ or $[R: I]<l$. In the latter case $J \subseteq I$, so $b_{n} \equiv a_{n} \equiv a_{m} \equiv b_{m} \bmod I$. Now suppose $n \not \equiv m \bmod [R: I]$. Either $J \subseteq I$ or $I \subseteq J$. If $J \subseteq I$ then $b_{n} \equiv a_{n} \not \equiv a_{m} \equiv b_{m}$ $\bmod I$. If $I \subseteq J$ then $b_{n} \equiv a_{n} \not \equiv a_{m} \equiv b_{m} \bmod J$ (because $\left.0 \neq n-m<[R: J]\right)$, hence $b_{n} \not \equiv b_{m} \bmod I$. Homogeneity is shown similarly.

From now on, $R$ is always an infinite subring of a discrete valuation ring $R_{v}$. Note that the definitions of $\alpha_{v, R}(n)$ and $v$-sequence below depend only on $M_{v}$ and $R$, and thus do not distinguish between equivalent valuations.

2.4 Definition. A $v$-sequence for $R$ is an $\left\{M_{v}^{n} \cap R \mid n \in \mathbb{N}\right\}$-sequence in $R$. In other words, $\left(a_{n}\right)$ is a $v$-sequence for $R$ if and only if for all $n \in \mathbb{N}$ and all $i, j$,

$$
a_{i}-a_{j} \in M_{v}^{n} \quad \Longleftrightarrow \quad\left[R: M_{v}^{n} \cap R\right] \mid i-j
$$

and a homogeneous $v$-sequence if in addition, for all $n \in \mathbb{N}$ and all $j \geq 1$,

$$
a_{j} \in M_{v}{ }^{n} \quad \Longleftrightarrow \quad\left[R: M_{v}{ }^{n} \cap R\right] \mid j .
$$

If $\left[R: M_{v}{ }^{n} \cap R\right]$ is infinite, distinct elements of a $v$-sequence must be incongruent $\bmod M_{v}{ }^{n} \cap R$. Proposition 2.1 guarantees the existence of an infinite homogeneous $v$-sequence for every infinite subring $R$ of every discrete valuation ring $R_{v}$.

2.5 Definition. For $n \in \mathbb{N}_{0}, R$ an infinite subring of $R_{v}$ and $q \in \mathbb{N}$, let

$$
\alpha_{v, R}(n)=\sum_{j \geq 1}\left[\frac{n}{\left[R: M_{v}^{j} \cap R\right]}\right] \quad \text { and } \quad \alpha_{q}(n)=\sum_{j \geq 1}\left[\frac{n}{q^{j}}\right] .
$$

Infinite indices are allowed; $\frac{n}{\infty}=0$. Since $R$ is infinite, $\alpha_{v, R}(n)$ is always a finite number. We will frequently use the fact that $\alpha_{v, R}(n)>0$ if and only if $n \geq\left[R: M_{v} \cap R\right]$. If $Q$ is a prime ideal in a domain $D$, such that $D_{Q}$ is a discrete valuation ring, we write $v_{Q}$ for the corresponding valuation with value group $\mathbb{Z}$.

2.6 Facts. (i) If $Q$ is a prime ideal of finite index $q$ in $R$ such that $R_{Q}$ is a discrete valuation ring, then $\alpha_{v_{Q}, R}(n)=\alpha_{q}(n)$ for all $n$.

(ii) If $v$ is a discrete valuation, $R$ an infinite subring of $R_{v}$ and $v^{\prime}$ an extension of $v$ with $\left[\Gamma_{v^{\prime}}: \Gamma_{v}\right]=e$ finite, then $\alpha_{v^{\prime}, R}(n)=e \cdot \alpha_{v, R}(n)$ for all $n$.

Proof. (i) Since $Q$ is maximal, $\left(Q R_{Q}\right)^{n} \cap R=Q^{n}$ for all $n$. Using the fact that $Q$ contains a generator of $Q R_{Q}$ one sees that $\left[R: Q^{n}\right]=\left[R_{Q}:\left(Q R_{Q}\right)^{n}\right]=q^{n}$ for all $n$. (ii) For $k \in \mathbb{N}, M_{v^{\prime}}{ }^{k} \cap R=\left(M_{v^{\prime}}{ }^{k} \cap R_{v}\right) \cap R=M_{v}{ }^{\left\lceil\frac{k}{e}\right\rceil} \cap R$, where $\lceil x\rceil$ denotes the smallest integer greater or equal $x$. Each number $\left[\frac{n}{\left[R: M_{v}{ }^{j} \cap R\right]}\right]$ appears $e$ times, as $\left[\frac{n}{\left[R: M_{v^{\prime}}{ }^{k} \cap R\right]}\right]$ for $k=(j-1) e+1, \ldots, j e$, in the sum for $\alpha_{v^{\prime}, R}(n)$.

In the remainder of section two, $v$ is assumed to have value-group $\mathbb{Z}$. 
2.7 Lemma. Let $\left(a_{i}\right)_{i=1}^{n+1},\left(b_{i}\right)_{i=1}^{n}$ and $\left(c_{i}\right)_{i=1}^{n}$ be $v$-sequences for $R$, and $\left(c_{i}\right)_{i=1}^{n}$ homogeneous. Then

(a) $v\left(c_{1} \cdot \ldots \cdot c_{n}\right)=\alpha_{v, R}(n) \leq v\left(b_{1} \cdot \ldots \cdot b_{n}\right) \leq \alpha_{v, R}(n)+\max _{1 \leq i \leq n} v\left(b_{i}\right)$,

(b) $v\left(\prod_{i=1}^{n}\left(a_{n+1}-a_{i}\right)\right)=\alpha_{v, R}(n) \leq v\left(\prod_{i=1}^{n}\left(r-b_{i}\right)\right)$ for all $r \in R$.

Proof. $v\left(c_{1} \cdot \ldots \cdot c_{n}\right)=\sum_{j \geq 1}\left|\left\{i \mid 1 \leq i \leq n, v\left(c_{i}\right) \geq j\right\}\right|$ and similarly for the $b_{i}$. Since for finite index $M_{v}{ }^{j} \cap R$ every $\left[R: M_{v}{ }^{j} \cap R\right]$ successive terms of a $v$-sequence form a complete residue system of $R \bmod M_{v}{ }^{j} \cap R$, we have $\forall j \in \mathbb{N}$

$$
\left|\left\{i \mid v\left(c_{i}\right) \geq j\right\}\right|=\left[\frac{n}{\left[R: M_{v}^{j} \cap R\right]}\right] \leq\left|\left\{i \mid v\left(b_{i}\right) \geq j\right\}\right| \leq\left[\frac{n}{\left[R: M_{v}^{j} \cap R\right]}\right]+1 .
$$

This implies (a) (and, since the 1 on the right can only occur if $\left[R: M_{v}{ }^{j} \cap R\right] \not n$, $v\left(b_{1} \cdot \ldots \cdot b_{n}\right) \leq \alpha_{v, R}(n)+\max _{1 \leq i \leq n} v\left(b_{i}\right)-\max \left\{j \mid\left[R: M_{v}{ }^{j} \cap R\right]\right.$ divides $\left.\left.n\right\}\right)$. By Fact 2.2 (ii) about $\mathcal{I}$-sequences, (b) is a special case of (a).

2.8 Theorem. Let $R$ be an infinite subring of $R_{v}$. An $R_{v}$-basis of $\operatorname{Int}\left(R, R_{v}\right)$ is given by

$$
f_{0}=1 \quad \text { and } \quad f_{n}(x)=\frac{\prod_{i=1}^{n}\left(x-a_{i}\right)}{\prod_{i=1}^{n}\left(a_{n+1}-a_{i}\right)} \quad(n \geq 1),
$$

where $\left(a_{n}\right)_{n=1}^{\infty}$ is a v-sequence for $R$.

Proof. An infinite $v$-sequence $\left(a_{n}\right)_{n=1}^{\infty}$ in $R$ exists by Proposition 2.1 applied to $\left\{M_{v}{ }^{n} \cap R \mid n \in \mathbb{N}\right\}$. The $f_{n}$, being a $K$-basis of $K[x]$, are free generators of the $R_{v}$-module they generate in $K[x]$, call this module $F$. Since by Lemma 2.7 every $f_{n}$ maps $R$ to $R_{v}, F \subseteq \operatorname{Int}\left(R, R_{v}\right)$. For the reverse inclusion we show the stronger statement that $\operatorname{Int}\left(A, R_{v}\right) \subseteq F$, where $A=\left\{a_{n} \mid n \in \mathbb{N}\right\}$. Let $f \in \operatorname{Int}\left(A, R_{v}\right), f=$ $\sum_{j=0}^{N} l_{j} f_{j}$ with $l_{j} \in K$. We show inductively that the $l_{j}$ are in $R_{v} . l_{0}=f\left(a_{1}\right) \in R_{v}$. The induction hypothesis is $l_{j} \in R_{v}$ for $0 \leq j<n$. Using this and the facts that $f_{j}\left(a_{i}\right)=0$ for $j \geq i$ and $f_{j}\left(a_{j+1}\right)=1$, we see that $f\left(a_{n+1}\right)=l_{n}+\sum_{j=0}^{n-1} l_{j} f_{j}\left(a_{n+1}\right)$. Since $f_{j}\left(a_{i}\right) \in R_{v}$ for all $i, j$ (by Lemma 2.7) and $f \in \operatorname{Int}\left(A, R_{v}\right)$, the sum on the right as well as $f\left(a_{n+1}\right)$ is in $R_{v}$, therefore $l_{n} \in R_{v}$.

Remark. For an infinite subring $R$ of $R_{v}$ and $A \subseteq R$, the proof of Theorem 2.8 shows that if $A$ contains an infinite $v$-sequence for $R$, then $\operatorname{Int}\left(A, R_{v}\right)=\operatorname{Int}\left(R, R_{v}\right)$. The converse holds, too (the criterion for $\operatorname{Int}\left(A, R_{v}\right)=\operatorname{Int}\left(R, R_{v}\right)$ in [7] is easily seen to be equivalent to $A$ containing an infinite $v$-sequence for $R$ ).

Corollary 1. $\alpha_{v, R}(n)=\max \left\{\min _{r \in R} v(f(r)) \mid f\right.$ monic $\left.\in K[x], \operatorname{deg} f=n\right\}$ and $M_{v}^{-\alpha_{v, R}(n)}=\left\{\right.$ leading coefficients of $n$-th degree polynomials in $\left.\operatorname{Int}\left(R, R_{v}\right)\right\} \cup\{0\}$.

Proof. The second statement can be read off the theorem using Lemma 2.7 (b); the first one then follows by Theorem 1.2.

Pólya's Satz IV [16] is a special case: if $P$ is a prime ideal in a domain $R$ such that $R_{P}$ is a discrete valuation $\operatorname{ring}$ and $[R: P]=q$, then (by Proposition 1.4 with $B=R$ and Fact 2.6 i) $\alpha_{q}(n)=\max \left\{\min _{r \in R} v_{P}(f(r)) \mid f \in R[x] \backslash P[x], \operatorname{deg} f=n\right\}$.

Corollary 2. Let $g_{n}(x)=\prod_{i=1}^{n}\left(x-a_{i}{ }^{(n)}\right)$, where $\left(a_{i}^{(n)}\right)_{i=1}^{n}$ is a v-sequence for $R$ when $n \geq\left[R: M_{v} \cap R\right]$, and let $g_{n}$ be any monic polynomial in $R_{v}[x]$ of degree $n$ 
for $0 \leq n<\left[R: M_{v} \cap R\right]$. Further, for $n \in \mathbb{N}_{0}$, let $c_{n} \in K$ with $v\left(c_{n}\right)=-\alpha_{v, R}(n)$. Then $\left(c_{n} g_{n}\right)_{n \in \mathbb{N}_{0}}$ is an $R_{v}$-basis of $\operatorname{Int}\left(R, R_{v}\right)$.

Proof. For all $n \in \mathbb{N}_{0}, r \in R, \quad v\left(g_{n}(r)\right) \geq \alpha_{v, R}(n)$ (by Lemma 2.7, in case $n \geq$ $\left[R: M_{v} \cap R\right]$, and because $g_{n} \in R_{v}[x]$ and $\alpha_{v, R}(n)=0$ otherwise). By the maximality of $\alpha_{v, R}(n)$ (Corollary 1), $\min _{r \in R} v\left(g_{n}(r)\right)=\alpha_{v, R}(n)$. Therefore $\left(c_{n} g_{n}\right)_{n \in \mathbb{N}_{0}}$ is an $R_{v}$-basis of $\operatorname{Int}\left(R, R_{v}\right)$ by Corollary 1 and Theorem 1.2 (ii).

\section{Polynomials mapping a SUbring into a Krull Ring}

Notation. Let $S$ be a domain with quotient field $K$, such that $S=\bigcap_{v \in \mathcal{V}} R_{v}, \mathcal{V}$ a set of discrete valuations (with value-group $\mathbb{Z}$ ) on $K$; and $R$ an infinite subring of $S$. We put $I_{n}=\{$ leading coefficients of $n$-th degree polynomials in $\operatorname{Int}(R, S)\} \cup\{0\}$ and introduce names for recurring additional conditions:

(F) $\forall q \in \mathbb{N}\left\{Q \unlhd R \mid[R: Q]=q\right.$ and $Q=M_{v} \cap R$ for some $\left.v \in \mathcal{V}\right\}$ is a finite set.

(C) For every prime ideal $Q$ of finite index in $R$, the set of $M_{v}{ }^{n} \cap R$ with $n \in \mathbb{N}$, $v \in \mathcal{V}$, and $M_{v} \cap R=Q$, if not empty, forms a descending chain of ideals.

Note that $(\mathrm{C})$ holds naturally in two cases: when there is only one $M_{v}$ such that $M_{v} \cap R=Q$, and when every $M_{v}{ }^{n} \cap R$ with $M_{v} \cap R=Q$ is a power of $Q$.

3.0 Lemma (Cahen [4]). If $R$ is an infinite subring of a Krull ring $S$ and $q \in \mathbb{N}$, then $S$ has at most finitely many height 1 prime ideals $P$ with $[R: P \cap R]=q$.

Proof. There exists $r \in R$ with $r^{q}-r \neq 0$. For every $P$ with $Q=R \cap P$ of index $q$ in $R, r^{q}-r \in Q \subseteq P$, so the statement follows by the definition of Krull ring.

3.1 Lemma. Let $v \in \mathcal{V}$ be such that $M_{v} \cap R=Q \neq(0)$, and $L$ the quotient field of $R$. If $R_{Q}$ is a valuation ring, then it is a discrete valuation ring and $R_{Q}=R_{v} \cap L$. If $Q$ is also a maximal ideal, then, for every $n \in \mathbb{N}, M_{v}^{n} \cap R$ is a power of $Q$.

Proof. For any valuation ring $V$ with quotient field $L$ and maximal ideal $M$ we have $L \backslash V=\left\{r \in L^{*} \mid r^{-1} \in M\right\}$. Put $R_{v} \cap L=R_{w}$ and $M_{v} \cap L=M_{w}$; then $R_{w}$ and $R_{Q}$ are valuation rings with quotient field $L$ and maximal ideals $M_{w}$ and $Q R_{Q}$, respectively. $R \subseteq R_{w}$ and $M_{w} \cap R=M_{v} \cap R=Q$ imply $R_{Q} \subseteq R_{w}$ and also $Q R_{Q} \subseteq M_{w}$. By the latter inclusion $L \backslash R_{Q}=\left\{r \in L^{*} \mid r^{-1} \in Q R_{Q}\right\} \subseteq$ $\left\{r \in L^{*} \mid r^{-1} \in M_{w}\right\}=L \backslash R_{w}$. This shows $R_{Q}=R_{w}=R_{v} \cap L$, so $R_{Q}$ is a discrete valuation ring and every $M_{v}^{n} \cap R_{Q}$ is a power of $Q R_{Q}$. If $Q$ is maximal, then $\left(Q R_{Q}\right)^{k} \cap R=Q^{k}$ for all $k$, so $M_{v}^{n} \cap R$ is a power of $Q$.

3.2 Lemma. (C) implies: For every finite set $\mathcal{M}$ of prime ideals of finite index in $R$ and every $m \in \mathbb{N}$, there exists a sequence $\left(a_{i}\right)_{i=0}^{m}$ in $R$ that is a homogeneous $v$-sequence for all $v$ in $\mathcal{V}$ with $M_{v} \cap R \in \mathcal{M}$, simultaneously.

Proof. For every $Q \in \mathcal{M}, \mathcal{I}_{Q}=\left\{M_{v}{ }^{n} \cap R \mid v \in \mathcal{V}, n \in \mathbb{N}, M_{v} \cap R=Q\right\}$ (if not empty) is a descending chain by $(\mathrm{C})$, so there exists a homogeneous $\mathcal{I}_{Q}$-sequence $\left(a_{i}{ }^{(Q)}\right)_{i=0}^{\infty}$ in $R$ by Proposition 2.1. For each $Q$ with $\mathcal{I}_{Q} \neq \emptyset$ let $I_{Q}$ be an element of $\mathcal{I}_{Q}$ with $\left[R: I_{Q}\right]>m$. $I_{Q}=M_{v}{ }^{n} \cap R$ for some $v$ and $n$, and therefore it contains $Q^{n}$. Since different $Q$ are co-prime, there exists, by the Chinese Remainder Theorem, a sequence $\left(a_{i}\right)_{i=0}^{m}$ in $R$ that is congruent to $\left(a_{i}{ }^{(Q)}\right)_{i=0}^{m}$ modulo $I_{Q}$ for all $Q \in \mathcal{M}$. By Lemma 2.3, this a homogeneous $\mathcal{I}_{Q}$-sequence for all $Q \in \mathcal{M}$, i.e., a homogeneous $v$-sequence for all $v$ with $M_{v} \cap R \in \mathcal{M}$. 
From Lemma 3.0, Lemma 3.1 and the fact that the powers of an ideal $Q$ form a descending sequence, we conclude that the hypothesis of Theorem 3.4 below is satisfied in at least one natural setting:

3.3 Fact. If $S$ is a Krull ring, $\mathcal{V}=\left\{v_{P} \mid P \in \operatorname{Spec}^{1}(S)\right\}$, and $R$ an infinite subring such that $R_{Q}$ is a valuation ring for every finite index $Q=P \cap R, P \in \operatorname{Spec}^{1}(S)$, then $(\mathrm{C})$ and $(\mathrm{F})$ both hold.

In the following theorem, the case where $S$ is a Dedekind ring and $R=S$ is due to Cahen [4] (also pertinent: [5]).

3.4 Theorem. Let $R$ be an infinite subring of $S=\bigcap_{v \in \mathcal{V}} R_{v}$. If $(\mathrm{C})$ and $(\mathrm{F})$ hold, then

$$
I_{n}=\bigcap_{v \in \mathcal{V}} M_{v}^{-\alpha_{v, R}(n)} \quad\left(n \in \mathbb{N}_{0}\right)
$$

and there exists a regular sequence of monic polynomials $\left(g_{n}\right)$ in $R[x]$ such that

$$
\operatorname{Int}(R, S)=\sum_{n \geq 0} I_{n} g_{n}
$$

namely, $g_{n}(x)=\prod_{i=1}^{n}\left(x-a_{i}{ }^{(n)}\right)$, where $\left(a_{i}{ }^{(n)}\right)_{i=1}^{n}$ is a simultaneous $v$-sequence for all $v \in \mathcal{V}$ with $\left[R: M_{v} \cap R\right] \leq n$.

Proof. Int $\left(R, \bigcap_{v \in \mathcal{V}} R_{v}\right)=\bigcap_{v \in \mathcal{V}} \operatorname{Int}\left(R, R_{v}\right)$, therefore $I_{n} \subseteq \bigcap_{v \in \mathcal{V}} M_{v}{ }^{-\alpha_{v, R}(n)}$ (by Theorem 2.8, Corollary 1). For the reverse inclusion, let $c \in \bigcap_{v \in \mathcal{V}} M_{v}^{-\alpha_{v}(n)}$. Set $\mathcal{V}_{n}=\left\{v \in \mathcal{V} \mid \alpha_{v, R}(n)>0\right\}=\left\{v \in \mathcal{V} \mid\left[R: M_{v} \cap R\right] \leq n\right\} ;$ then $\left\{M_{v} \cap R \mid v \in \mathcal{V}_{n}\right\}$ is finite by $(\mathrm{F})$. Let $\left(a_{i}{ }^{(n)}\right)_{i=1}^{n}$ in $R$ be a homogeneous $v$-sequence for all $v \in \mathcal{V}_{n}$ simultaneously (which exists by Lemma 3.2) and $g_{n}(x)=\prod_{i=1}^{n}\left(x-a_{i}{ }^{(n)}\right)$. Then $\min _{r \in R} v(g(r)) \geq \alpha_{v, R}(n)$ for all $v \in \mathcal{V}$ (by Lemma 2.7 when $v \in \mathcal{V}_{n}$, and because $\alpha_{v, R}(n)=0$ and $g_{n} \in R[x]$ otherwise), which means $c g(x) \in \operatorname{Int}\left(R, \bigcap_{v \in \mathcal{V}} R_{v}\right)$ and hence $c \in I_{n}$. This completes the proof of the first statement and also shows, for all $n \geq 0$, that $I_{n} g_{n} \subseteq \operatorname{Int}\left(R, \bigcap_{v \in \mathcal{V}} R_{v}\right)$, so the second follows by Lemma 0.1.

From now on, $S$ is a Krull ring. By convention, the empty intersection or product of ideals of $S$ equals $S$. We denote the set of height 1 prime ideals of $S$ by $\operatorname{Spec}^{1}(S)$ or $\mathcal{P}$. If $P \in \mathcal{P}$, we write $\alpha_{P, R}$ for $\alpha_{v_{P}, R}$ and, if $j \in \mathbb{N}_{0}, P^{(j)}$ for $\left(P S_{P}\right)^{j} \cap S$. With this notation we have, for $n \in \mathbb{N}_{0}$ and $P \in \mathcal{P}$ :

$$
\alpha_{P, R}(n)=\sum_{j \geq 1}\left[\frac{n}{\left[R: P^{(j)} \cap R\right]}\right] .
$$

3.5 Lemma. Let $S$ be a Krull ring and $\mathcal{V}=\left\{v_{P} \mid P \in \mathcal{P}\right\}$. If $(\mathrm{C})$ holds, then $\operatorname{Int}(R, S)$ has a regular basis if and only if $\bigcap_{P \in \mathcal{P},[R: P \cap R] \leq n} P^{\left(\alpha_{P, R}(n)\right)}$ is principal for all $n$.

Proof. $\alpha_{P, R}(n) \neq 0$ if and only if $[R: P \cap R] \leq n$. Since (F) holds by Lemma 3.0, this only happens for finitely many $P$ for each $n$. If $\left\{a_{P} \mid P \in \mathcal{P}\right\}$ is a set of integers, only finitely many of them non-zero, then $\bigcap_{P \in \mathcal{P}}\left(P S_{P}\right)^{-a_{P}}$ is principal if and only if $\bigcap_{P \in \mathcal{P}}\left(P S_{P}\right)^{a_{P}}$ is, namely if there exists $c \in K$ with $v_{P}(c)=a_{P}$ for all $P \in P$. If all $a_{P}$ are non-negative then $\bigcap_{P \in \mathcal{P}}\left(P S_{P}\right)^{a_{P}}=\bigcap_{a_{P}>0} P^{\left(a_{P}\right)}$. Applied to $\bigcap_{P \in \mathcal{P}}\left(P S_{P}\right)^{-\alpha_{P, R}(n)}$, which is $I_{n}$ by Theorem 3.4, with Lemma 0.1 (iii) in mind, this proves the claim. 
3.6 Theorem. Let $R$ be an infinite subring of a Krull ring $S, \mathcal{P}=\operatorname{Spec}^{1}(S)$, $\mathcal{P}^{*}=\{P \in \mathcal{P} \mid[R: P \cap R]$ finite $\}$ and $\mathcal{Q}=\left\{R \cap P \mid P \in \mathcal{P}^{*}\right\}$. If $R_{Q}$ is a valuation ring for all $Q \in \mathcal{Q}$, then $R_{Q}$ is a discrete valuation ring for all $Q \in \mathcal{Q}$ and

$\operatorname{Int}(R, S)$ has a regular basis $\Longleftrightarrow \forall q \in \mathbb{N} \bigcap_{\substack{P \in \mathcal{P} \\[R: R \cap P]=q}} P^{\left(e_{P}\right)}$ is a principal ideal of $S$, where $e_{P}$ is the ramification index of $P S_{P}$ over $Q R_{Q}$, for $P \in \mathcal{P}^{*}, Q=P \cap R$.

Proof. Let $\mathcal{P}_{q}=\{P \in \mathcal{P} \mid[R: P \cap R]=q\}, P \in \mathcal{P}_{q}, Q=P \cap R, L$ the quotient field of $R$; then by Lemma 3.1 $R_{Q}=S_{P} \cap L$ and $R_{Q}$ is a discrete valuation ring. $v_{P}^{\prime}=\left(1 / e_{P}\right) v_{P}$ is equivalent to $v_{P}$ and is an extension of $v_{Q}$ to $K$ with $\left[\Gamma_{v_{P}^{\prime}}: \Gamma_{v_{Q}}\right]=$ $e_{P}$. By the Facts 2.6 (ii) and (i), $\alpha_{P, R}(n)=\alpha_{v_{P}^{\prime}, R}(n)=e_{P} \alpha_{Q, R}(n)=e_{P} \alpha_{q}(n)$.

If we call the left and right sides of the claimed equivalence (l) and (r), re-

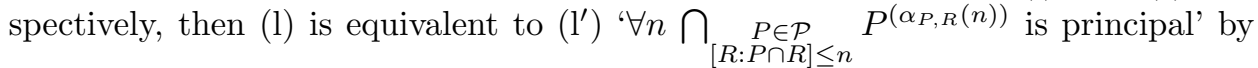
Lemma 3.5 (whose condition (C) holds by Fact 3.3). We know that

$$
\bigcap_{\substack{P \in \mathcal{P} \\[R: P \cap R] \leq n}} P^{\left(\alpha_{P, R}(n)\right)}=\bigcap_{q \leq n} \bigcap_{P \in \mathcal{P}_{q}} P^{\left(e_{P} \alpha_{q}(n)\right)} .
$$

The latter is clearly principal provided all $\bigcap_{P \in \mathcal{P}_{q}} P^{\left(e_{P}\right)}$ are; thus $(\mathrm{r}) \Rightarrow\left(\mathrm{l}^{\prime}\right)$.

For $(\mathrm{l}) \Rightarrow(\mathrm{r})$, suppose $\bigcap_{q \leq n} \bigcap_{P \in \mathcal{P}_{q}} P^{\left(e_{P} \alpha_{q}(n)\right)}=s_{n} S$ for all $n$. We see that $s_{q} S=\bigcap_{P \in \mathcal{P}_{q}} P^{\left(e_{P}\right)} \cap \bigcap_{l<q} \bigcap_{P \in \mathcal{P}_{l}} P^{\left(e_{P} \alpha_{l}(q)\right)}$, because $\alpha_{q}(q)=1$. This allows an induction on $q$ : from the formula for $s_{q} S$ we conclude that $\bigcap_{P \in \mathcal{P}_{q}} P^{\left(e_{P}\right)}$ is principal if $\bigcap_{P \in \mathcal{P}_{l}} P^{\left(e_{P}\right)}$ is principal for all $l<q$.

Corollary 1. If $R \subseteq S$ is an extension of Krull rings such that ht $(P \cap R) \leq 1$ for all height 1 prime ideals $P$ of $S$, then

$$
\operatorname{Int}(R, S) \text { has a regular basis } \Longleftrightarrow \forall q \in \mathbb{N} \bigcap_{\substack{Q \in \operatorname{Spec}^{1}(R) \\[R: Q]=q}} \operatorname{div}(Q S) \text { is principal, }
$$

where $\operatorname{div}(Q S)$ means the smallest divisorial ideal containing $Q S$.

Proof. If $R \subseteq S$ is an extension of Krull rings with the stated property and $Q$ is in $\operatorname{Spec}^{1}(R)$, then $\operatorname{div}(Q S)=\bigcap_{P \in \operatorname{Spec}^{1}(S)} P^{\left(e_{P}\right)}$, where $e_{P}=e(P \mid Q)$ is the ramification index of $P S_{P}$ over $Q R_{Q}$ [1, p. 183].

In particular, if $R \subseteq S$ is an extension of Dedekind rings, then

$$
\operatorname{Int}(R, S) \text { has a regular basis } \Longleftrightarrow \forall q \in \mathbb{N} \prod_{\substack{Q \in \operatorname{Spec}(R) \\[R: Q]=q}} Q S \text { is principal. }
$$

A different specialization gives Ostrowski's criterion [15]. If $S$ is a Krull ring,

$$
\operatorname{Int}(S) \text { has a regular basis } \Longleftrightarrow \forall q \in \mathbb{N} \prod_{\substack{P \in \operatorname{Spec}^{1}(S) \\[S: P]=q}} P \text { is principal. }
$$

When a regular basis exists, we can give a fairly explicit description of one. (For $\operatorname{Int}(S), S$ a Dedekind ring, there also is a different construction by Gerboud [9].) 
Corollary 2. In the situation of Theorem 3.6, if $\bigcap_{[R: P \cap R]=q} P^{\left(e_{P}\right)}=c_{q} S(q \in \mathbb{N})$ then a regular basis of $\operatorname{Int}(R, S)$ is given by $f_{0}=1$,

$$
f_{n}(x)=\prod_{q \leq n} c_{q}^{-\alpha_{q}(n)} \prod_{i=1}^{n}\left(x-a_{i}^{(n)}\right) \quad(n \in \mathbb{N})
$$

where $\left(a_{i}{ }^{(n)}\right)_{i=1}^{n} \subseteq R$ is a $v_{P}$-sequence for all $P \in \mathcal{P}$ with $[R: P \cap R] \leq n$.

Proof. $v_{P}\left(c_{q}^{-\alpha_{q}(n)}\right)=-e_{P} \alpha_{q}(n)=-\alpha_{P, R}(n)$ for the $P \in \mathcal{P}$ with $[R: P \cap R]=q$, and zero for all other $P \in \mathcal{P}$, so $v_{P}\left(\prod_{q \leq n} c_{q}^{-\alpha_{q}(n)}\right)=-\alpha_{P, R}(n)$ for all $P \in \mathcal{P}$ (since $\alpha_{P, R}(n)=0$ if $\left.n<[R: P \cap R]\right)$. Therefore the $f_{n}$ are an $S_{P}$-basis of $\operatorname{Int}\left(R, S_{P}\right)$ for all $P \in \mathcal{P}$ simultaneously, by Theorem 2.8, Corollary 2 .

\section{REFERENCES}

1. S. Balcerzyk and T. Józefiak, Commutative Noetherian and Krull Rings, Ellis Horwood, Chichester; distr. by Wiley, New York, 1989. MR 92f:13001

2. P.-J. Cahen, Integer-valued polynomials on a subset, Proc. Amer. Math. Soc. 117 (1993), 919-929. MR 93e:13011

3. P.-J. Cahen, Parties pleines d'un anneau noethérien, J. Algebra 157 (1993), 199-212. MR 94d:13021

4. P.-J. Cahen, Polynômes à valeurs entières, Canad. J. Math. 24 (1972), 747-754. MR 46:9027

5. P.-J. Cahen and J.-L. Chabert, Coefficients et valeurs d'un polynôme, Bull. Sci. Math. $2^{e}$ Sér. 95 (1971), 295-304. MR 45:5126

6. J.-L. Chabert and G. Gerboud, Polynômes à valeurs entières et binômes de Fermat, Canad. J. Math. 45 (1993), 6-21. MR 94c:13020

7. S. Frisch, Substitution and Closure of Sets under Integer-valued Polynomials, J. Number Theory 56 (1996), 396-403.

8. G. Gerboud, Substituabilité d'un anneau de Dedekind, CR Acad. Sci. Paris 317 (1993), 29-32. MR 94e: 13039

9. G. Gerboud, Construction, sur un anneau de Dedekind, d'une base régulière de polynômes à valeurs entières, manuscripta math. 65 (1989), 167-179. MR 90h:13016

10. R. Gilmer, Prüfer Domains and Rings of Integer-Valued Polynomials, J. Algebra 129 (1990), 502-517. MR 91b:13023

11. R. Gilmer, Sets That Determine Integer-Valued Polynomials, J. Number Theory 33 (1989), 95-100. MR 90g:11142

12. H. Gunji and D. L. McQuillan, On a Class of Ideals in an Algebraic Number Field, J. Number Theory 2 (1970), 207-222. MR 41:1681

13. D. L. McQuillan, On a Theorem of R. Gilmer, J. Number Theory 39 (1991), 245-250. MR 92i: 13016

14. D. L. McQuillan, On Prüfer domains of polynomials, J. Reine Angew. Math. 358 (1985), 162-178. MR 86k:13019

15. A. Ostrowski, Über ganzwertige Polynome in algebraischen Zahlkörpern, J. Reine Angew. Math. 149 (1919), 117-124.

16. G. Pólya, Über ganzwertige Polynome in algebraischen Zahlkörpern, J. Reine Angew. Math. 149 (1919), 97-116.

Institut für Mathematik C, Technische Universität Graz, Kopernikusgasse 24, A8010 Graz, Austria

E-mail address: frisch@blah.math.tu-graz.ac.at 